\title{
Electroluminescence on-off ratio control of $n-i-n$ GaAs/AlGaAs-based resonant tunneling structures
}

\author{
E. R. Cardozo de Oliveira, ${ }^{1,2}$ A. Pfenning, ${ }^{2}$ E. D. Guarin Castro, ${ }^{1}$ M. D. Teodoro, ${ }^{1,}$ E. C. $\operatorname{dos}$ Santos, ${ }^{1}$ V. Lopez-Richard, ${ }^{1}$ \\ G. E. Marques, ${ }^{1}$ L. Worschech, ${ }^{2}$ F. Hartmann, ${ }^{2}$ and S. Höfling ${ }^{2,3}$ \\ ${ }^{1}$ Departamento de Física, Universidade Federal de São Carlos, 13565-905 São Carlos, São Paulo, Brazil \\ ${ }^{2}$ Technische Physik, Physikalisches Institut, Röntgen Center for Complex Material Systems (RCCM), Universität Würzburg, Am Hubland, \\ D-97074 Würzburg, Germany \\ ${ }^{3}$ SUPA, School of Physics and Astronomy, University of St. Andrews, St. Andrews KY16 9SS, United Kingdom
}

(Received 18 June 2018; revised manuscript received 5 July 2018; published 1 August 2018)

\begin{abstract}
We explore the nature of the electroluminescence (EL) emission of purely $n$-doped GaAs/AlGaAs resonant tunneling diodes (RTDs) and the EL evolution with voltage. A singular feature of such a device is unveiled when the electrical output current changes from higher to lower values and the EL on-off ratio is enhanced by two orders of magnitude compared to the current on-off ratio. By combining the EL and the current properties, we are able to identify two independent impact ionization channels associated with the coherent resonant tunneling current and the incoherent valley current. We also perform the same investigation with an associated series resistance, which induces a bistable electrical output in the system. By simulating a resistance variation for the current voltage and the EL, we are able to tune the EL on-off ratio by up to six orders of magnitude. We further observe that the EL on and off states can be either direct or inverted compared to the tunneling current of the on and off states. This electroluminescence, combined with the unique RTD properties, such as the negative differential resistance and high-frequency operation, enables the development of high-speed functional optoelectronic devices and optical switches.
\end{abstract}

DOI: 10.1103/PhysRevB.98.075302

\section{INTRODUCTION}

The transport signature of resonant tunneling diodes (RTDs), that is, a peak current density followed by a region of negative differential resistance (NDR) [1,2], enables RTDs to be exploited in a broad range of applications, such as terahertz oscillators, high-speed logic gates, light detectors, and thermometers [3-6]. Although the heterostructure device layouts of RTDs are rather simple, certain requirements must be achieved in order to produce high quality devices, e.g., low leakage current and large peak-to-valley current ratio (PVCR) [7]. Apart from being the fastest semiconductor devices used for practical applications [8,9], RTDs are also suitable for optoelectronic uses as they can be light emitters and detectors $[5,10,11]$. In purely $n$-doped RTDs, light emission occurs via electroluminescence (EL) due to either impact ionization processes taking place along the structure $[10,12,13]$ or Zener tunneling, that is, direct interband tunneling from the valence to conduction band under high electric fields $[14,15]$. Light emission enables the extraction of reliable information about the diode, such as charge buildup, carrier dynamics, and system temperature, all of which enrich the whole transport picture [16-18]. Since the electroluminescence is an intrinsic response of the RTD to electrical excitation, it provides insights into the resonant tunneling process by revealing features of the transport mechanisms of the available charge carriers without the need for an external light source for electron-hole pair creation.

\footnotetext{
*Corresponding author: mdaldin@df.ufscar.br
}

In this paper, we explore the coexistence of two transport channels in a purely $n$-doped RTD based on GaAs/AlGaAs and assess how they affect the emitted light by contributing independently to the impact ionization processes responsible for hole generation. The experimental observations have been theoretically simulated allowing for the corroboration of the hypothesis raised. We then engineer the EL emission configuration from monostable to bistable by tuning a resistance in series with the diode. We show that the optical on-off ratio can be larger than the electrical on-off ratio by three orders of magnitude and can be varied within six orders by changing the resistance in just one order of magnitude. This architecture can be a useful tool for developing high quality optical logic devices [19].

\section{SAMPLE AND EXPERIMENTAL SETUP}

The RTDs were grown by molecular beam epitaxy on top of a Si $n$-doped $\left(3 \times 10^{18}-\mathrm{cm}^{-3}\right) \mathrm{GaAs}$ substrate beginning with a 300 -nm $n$-doped $\left(3 \times 10^{18}-\mathrm{cm}^{-3}\right)$ GaAs buffer layer. The diode consists of a 100 -nm $n$-doped (from $3 \times 10^{18}$ - to $5 \times 10^{17}-\mathrm{cm}^{-3}$ ) GaAs layer, followed by an undoped region with two 20-nm-thick GaAs layers embedding two 3.5-nmthick $\mathrm{Al}_{0.6} \mathrm{Ga}_{0.4} \mathrm{As}$ barriers separated by a 4-nm-thick GaAs quantum well $(\mathrm{QW})$, forming the structure. The RTD is finalized by $100-\mathrm{nm}$ GaAs with a doping concentration of $n=2 \times 10^{17} \mathrm{~cm}^{-3}$ followed by a 300 -nm $n$-doped (from $2 \times 10^{17}$ - to $\left.3 \times 10^{18}-\mathrm{cm}^{-3}\right) \mathrm{Al}_{0.2} \mathrm{Ga}_{0.8}$ As layer and a 10 $\mathrm{nm}$-thick $n$-doped $\left(3 \times 10^{18}-\mathrm{cm}^{-3}\right) \mathrm{GaAs}$ capping layer. After the growth process, RTD mesas with diameters of $11 \mu \mathrm{m}$ were processed by electron-beam lithography in combination 


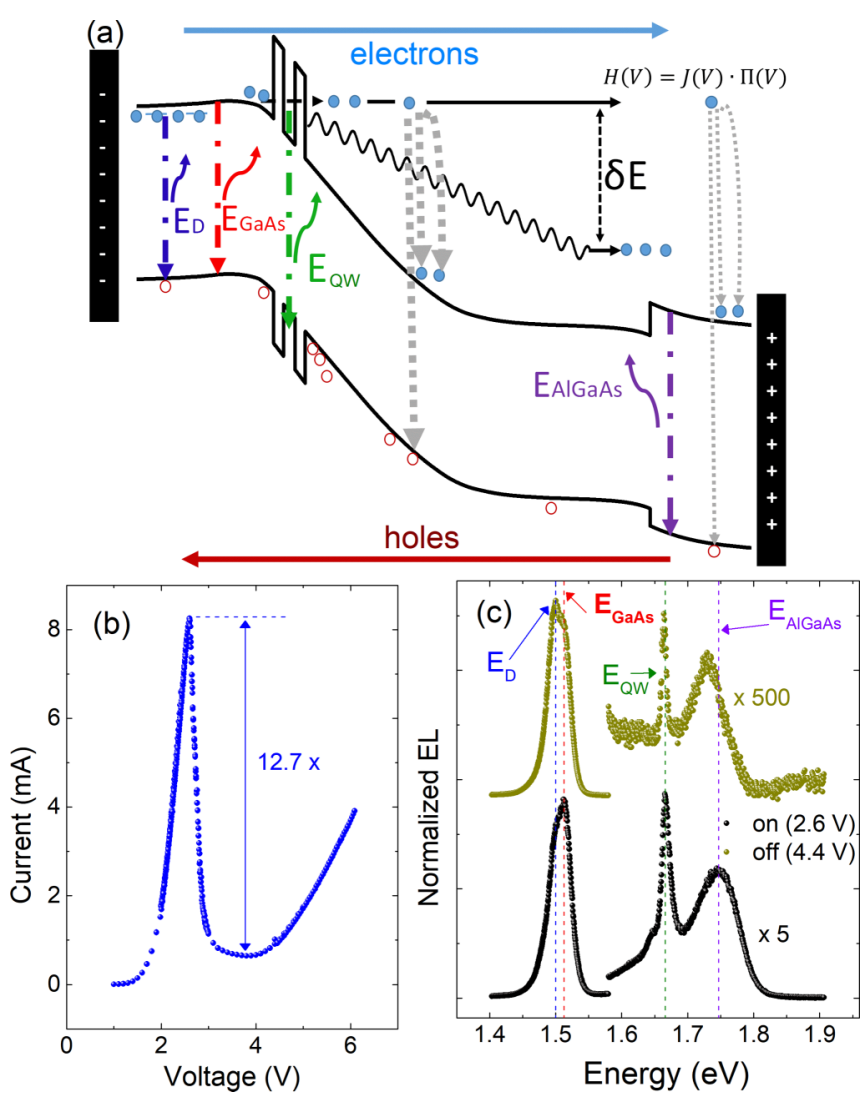

FIG. 1. (a) Numerical simulation of the band structure under forward bias. The blue (red) horizontal arrow shows the direction of electrons (holes) at this bias regime. Tunneling electrons undergo impact ionization processes either in the GaAs or in the $\mathrm{AlGaAs}$ regions (gray arrows with the ionization probability represented schematically through the arrow thicknesses), and the generated holes eventually recombine, so the region of each EL emission (vertical colored arrows) is shown schematically. (b) Measured $I-V$ characteristic at $T=10 \mathrm{~K}$. (c) Normalized EL spectra obtained at $2.6 \mathrm{~V}$ (black) and $4.4 \mathrm{~V}$ (dark yellow), respectively, before and after the resonance peak.

with dry chemical etching techniques. Figure 1(a) presents the numerically simulated band profile of the RTD under forward bias as well as the drift direction of electrons and holes with blue and red arrows, respectively.

Electrical and optical measurements were performed with the sample placed in a helium closed-cycle cryostat (AttocubeAttodry1000). All measurements presented in this paper were obtained at a temperature of $T=10 \mathrm{~K}$. The setup was used in combination with a confocal microscope (AttoCFMI) to carry out the electroluminescence experiments. The signal was collimated by an objective lens and transmitted along a multimode optical fiber, being dispersed by a $75-\mathrm{cm}$ spectrometer and detected by a silicon charge-coupled device detector (Andor-Shamrock/Idus).

\section{RESULTS AND DISCUSSION}

The $I-V$ characteristic is shown in Fig. 1(b). The peak current is $8.25 \mathrm{~mA}$ at $2.6 \mathrm{~V}$, and the PVCR is 12.7. During the impact ionization process, a conduction-band electron
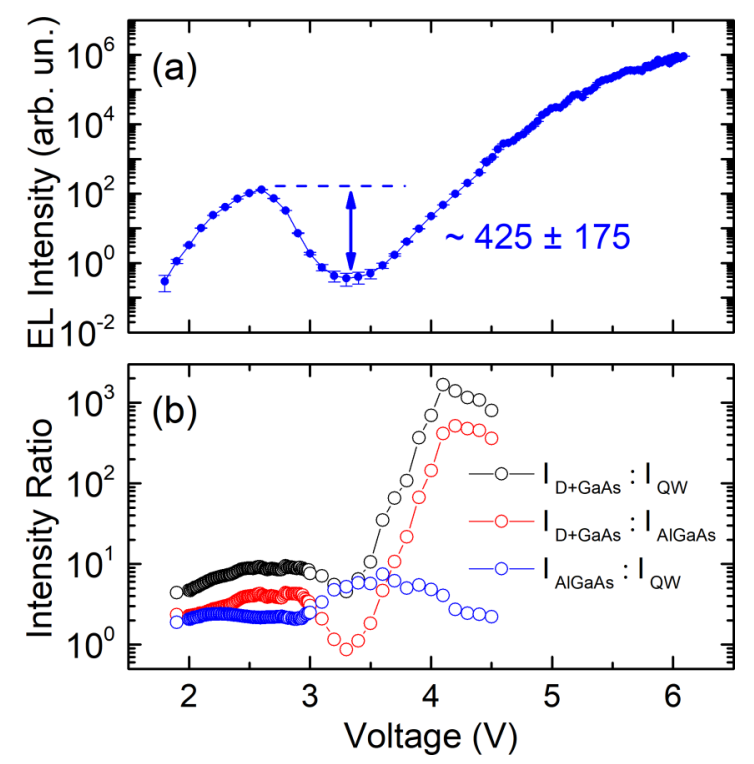

FIG. 2. (a) EL total intensity as function of voltage. (b) Voltagedependent intensity ratio between GaAs and QW (black), GaAs and AlGaAs (red), and AlGaAs and QW (blue) emissions.

with energy above the ionization threshold is scattered with a valence-band electron that is subsequently promoted to the conduction band leaving a hole in the valence band [Fig. 1(a)]. Depending on the spatial origin of the hole generation, the holes drift and recombine with electrons in different regions of the structure, e.g., close to the collector contact [10], in the QW, or in the emitter region. Figure 1(c) shows the normalized EL spectra obtained in two different voltage regimes before (black circles, bias voltage of $2.6 \mathrm{~V}$ ) and after (dark yellow circles, bias voltage of $4.4 \mathrm{~V}$ ) the resonant current peak. One can observe four distinct emission lines, which are schematically shown in Fig. 1(a) (dot-dashed arrows): $E_{\mathrm{D}}=1.500 \mathrm{eV}-$ associated with donor-band recombination, $E_{\mathrm{GaAs}}=1.512 \mathrm{eV}$ is the band-to-band emission in bulk GaAs, $E_{\mathrm{QW}}=1.664 \mathrm{eV}$ originates from the quantum well, and $E_{\mathrm{AlGaAs}}=1.746 \mathrm{eV}$ emerges from the AlGaAs region.

The QW and AlGaAs emission peaks are weaker compared to the $E_{\mathrm{D}}$ and $E_{\mathrm{GaAs}}$ lines, being $\sim 5 \times$ lower in the on-resonance case $(2.6 \mathrm{~V})$ and $\sim 500 \times$ lower off-resonance $(4.4 \mathrm{~V})$. For lower voltages, up to the resonance peak, the charge carrier density in the quantum well increases, leading to a higher recombination rate [20]. Afterwards, in the offresonance case, the charge carrier density in the quantum well is reduced, increasing the ratio between the bulk GaAs and the QW emissions. Additionally, after the current resonance (i.e., at $4.4 \mathrm{~V}$ ) the impact ionization in the GaAs layer after the double barrier is enhanced due to a high electric field in this region, leaving less electrons available to reach the $\mathrm{AlGaAs}$ layer with energies above the impact ionization threshold. The variation with voltage of the intensity integrated over the whole spectrum (EL- $V$ ) is presented in Fig. 2(a). The EL PVR is two orders of magnitude higher than the $I-V$ PVCR with an optical on-off ratio of approximately $425 \pm 175$.

The ratios between the donor emission line plus GaAs $\left(I_{\mathrm{D}+\mathrm{GaAs}}\right), \mathrm{AlGaAs}\left(I_{\mathrm{AlGaAs}}\right)$, and the $\mathrm{QW}\left(I_{\mathrm{QW}}\right)$ intensities are depicted in Fig. 2(b). They allow assessing the correlation 



FIG. 3. (a) EL intensity as a function of voltage. The gray circles represent the experimental data, and the red circles are the product of the current with the impact ionization rate calculated for GaAs. (b) Calculated $I-V$ characteristic, (c) Hole generation rate deduced from the calculated $I-V$. The inset in (b) represents the band structure considered in the model with a flatband for the barriers and the well.

between the available recombination channels. One can see that the ratio between $\mathrm{AlGaAs}$ and $\mathrm{QW}$ emissions is nearly constant (blue circles) and can be assumed as correlated as the voltage increases. In contrast, when compared with the GaAs emission, the ratio remains constant before the resonance but suffers a considerable increase after $3.5 \mathrm{~V}$ (black and red circles). This is evidence of a connection between the electrons localized at the resonant state in the QW with those contributing to the $\mathrm{AlGaAs}$ emission. The lack of data points beyond $4.5 \mathrm{~V}$ is due to limitations of the experimental setup.

Another peculiar feature observed in Fig. 2(a) is the exponential EL signal growth after the resonance, whereas the current in Fig. 1(b) shows a slow increase. As mentioned above, the main process for generating holes in this purely $n$-doped structure under applied bias is impact ionization, and thus the hole generation rate $H(V)$ should be proportional to both the current density $J(V)$ and the impact ionization rate $\Pi(V)$,

$$
H(V) \propto J(V) \Pi(V),
$$

with the ionization rate being described by the generalized Keldysh model [21],

$$
\Pi(V)=C\left(\frac{e V-E_{\mathrm{th}}}{E_{\mathrm{th}}}\right)^{a},
$$

where $C=93.659 \times 10^{10} \mathrm{~s}^{-1}$ and $a=4.743$ are derived parameters for GaAs and $E_{\text {th }}=1.8 \mathrm{eV}$ is the GaAs impact ionization threshold energy. Our observation matches well with the latter parameter since the structure starts emitting light from $1.8 \mathrm{~V}$ as one can see in Fig. 2(a). By using Eq. (1) with the measured current we obtained the hole generation rate, equivalent to the total EL intensity, which is presented in Fig. 3(a) as red circles together with the experimental data (gray circles). The hole generation rate is similar to the EL intensity before the resonance, however it fails from the NDR region and forward. At first glance, this could be ascribed to electric-field corrections not considered in Eq. (2). According to Ref. [21], when the system is under applied bias, the impact ionization model must be corrected, however the deviations occur at high electric fields and only for lower energies close to the threshold $E_{\mathrm{th}}$. Yet, in our case, the same field regime is achieved at higher energies (or voltages) where the models that include the electric field converge to Eq. (2), so the latter can be considered valid. Thus, a single transport channel contributing to the EL is proven to be inefficient to simulate the RTD emission, and it remains to prove that the presence of two impact ionization channels allows explaining this discrepancy.

In RTDs, the current can be divided into a coherent part $J_{\text {coh }}$, related to the resonant tunneling peak, and an incoherent part. The coherent current flowing through the double-barrier structure is simulated using the Tsu-Esaki equation for the density current, given by [1]

$$
\begin{aligned}
J_{\mathrm{coh}}= & \frac{e m^{*} k_{B} T}{2 \pi^{2} \hbar^{3}} \int_{0}^{\infty} T(E, V) \ln \\
& \times\left[\frac{1+\exp \left(\frac{E_{f}-E}{k_{B} T}\right)}{1+\exp \left(\frac{E_{f}-E-e V}{k_{B} T}\right)}\right] d E,
\end{aligned}
$$

where $e, m^{*}, k_{B}, T, \hbar, E, E_{f}, V$, and $T(E, V)$, respectively, are the electron charge, the effective electron mass of the material in the emitter, the Boltzmann constant, the temperature, the reduced Planck constant, the electron energy along the growth direction, the emitter Fermi level, the voltage necessary to obtain the inner tunneling transmission at the double barrier, and the transmission coefficient. The latter one is obtained by using the transfer-matrix method [22] and the band profile under an applied voltage as shown in the inset of Fig. 3(b). On the other hand, the incoherent (or excess) part $J_{\text {inco }}$ comprises other currents, such as hot electrons, thermal activation, and sidewall leakage [23-25]. Thermal activation can be discarded from this model as cryogenic temperatures suppress this process. The sidewall current is dependent on the perimeter of the structure, and for higher diameters this contribution becomes less relevant and can also be neglected (which is the case for a diameter of $11 \mu \mathrm{m}$ ). The excess current is then considered to be composed mostly by electrons with phase coherence and energy losses and can be accounted for by a standard exponential diode term [26], such as

$$
J_{\text {inco }}=h\left[\exp \left(\frac{\eta_{1} V}{k_{B} T}\right)-1\right],
$$

where $h$ is a fitting parameter and $\eta_{1}$ refers to the efficiency of the excess current.

Following Fig. 1(a), the coherent current consists of carriers tunneling through the double barrier without losing their phase coherence and energy as it is a resonant process, whereas the incoherent current consists of carriers tunneling sequentially into the double barrier, therefore losing their phase coherence and energy through scattering processes, such as phonon or lattice scattering. With this in mind, the coherent and incoherent EL channels, respectively, consist of

$$
H_{\mathrm{co}} \propto J_{\mathrm{co}} \Pi(V),
$$

and

$$
H_{\text {inco }} \propto J_{\text {inco }} \Pi(V-\delta E)
$$



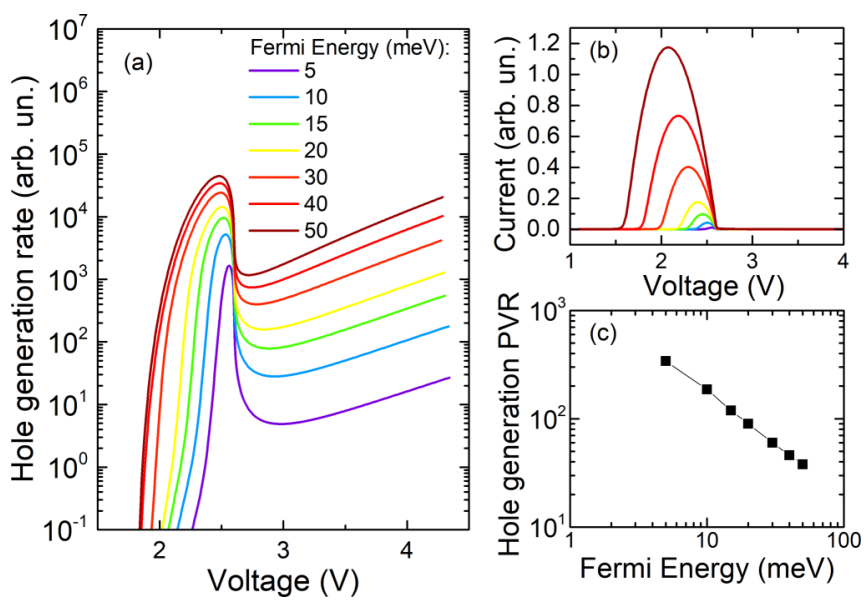

FIG. 4. (a) Hole generation rate calculated for different Fermi energy values. (b) Calculated coherent resonant tunneling current. (c) Hole generation PVR as function of Fermi energy.

with $\delta E$ being the energy loss due to the scattering processes. In a more general picture, a $\delta E$ term could also be added in the expression for the coherent channel, but as the EL emission starts exactly at the threshold energy $E_{\text {th }}=1.8 \mathrm{eV}$, we can assume that no energy loss take place for this channel.

When the coherent channel is off, the incoherent transport, described with Eq. (4), becomes dominant, prevailing in the hole generation for the external bias above $3.5 \mathrm{~V}$. With these assumptions and using $E_{f}=5 \mathrm{meV}$ and $T=10 \mathrm{~K}$, the calculations for the current are presented in Fig. 3(b) where, due to the completely coherent picture, the peak is narrower compared to the experiment. For the hole generation rate, the calculated curve shows good agreement with the EL intensity as displayed in Fig. 3(c). Thus, the calculated PVR for the hole generation rate is $\sim 340$, which coincides within the experimental error with the PVR displayed in Fig. 2(a).

Furthermore, a value of $\delta E=1.3 \mathrm{eV}$ was extracted by adjusting the incoherent channel according to the experiment. Under resonant conditions in RTDs, the contribution of coherent electrons prevails over the incoherent ones [25], and, according to Ref. [27], their mean free path in GaAs at an $n$-doping concentration of $1 \times 10^{17} \mathrm{~cm}^{-3}$ is $80 \mathrm{~nm}$. Thus we can assume that these coherent electrons are not likely to undergo any energy relaxation before the impact ionization event. On the other hand, after the resonance, charge carriers are more likely to experience lattice scattering as the probability of coherent transport drops, which reduces the mean free path and leads to the expected high-energy losses for the incoherent electrons. By considering the full picture of coherent and incoherent carriers, the correlation between QW and AlGaAs intensities, shown in Fig 2(b), can be understood as follows: Only electrons tunneling resonantly through the double barrier can reach the $\mathrm{AlGaAs}$ region for generating holes, whereas those subjected to sequential tunneling are not able to go further with energy higher than the impact ionization threshold. The same model was used to assess the effect of Fermi energy variation for a fixed temperature at $10 \mathrm{~K}$, and the results are shown in Fig. 4 for the completely coherent case. By increasing $E_{F}$, both the hole generation and the current increase as depicted in Figs. 4(a) and 4(b), respectively.

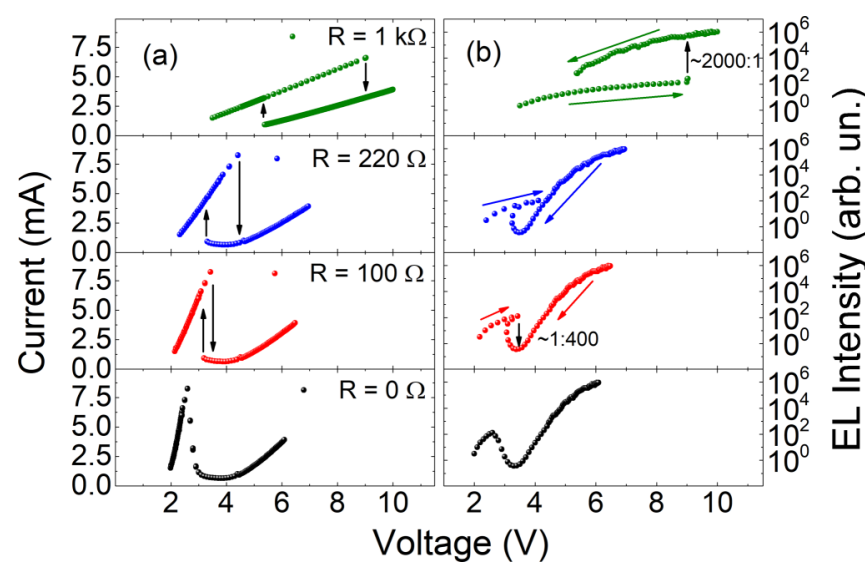

FIG. 5. (a) $I-V$ and (b) EL- $V$ characteristics. The data presented for $0-\Omega$ (black circles) and $1-\mathrm{k} \Omega$ (green circles) resistances are experimental whereas for 100 and $220 \Omega$, displayed as red and blue circles, respectively, are simulations using Kirchhoff's law.

However such an increase leads to an exponential reduction of the hole generation peak-to-valley ratio, displayed in Fig. 4(c).

Once the nature of the modulation of the light emission is understood, we can proceed and discuss how to engineer the optical response. A desirable feature in optical logic devices is the thorough control over the emission enhancement and decrease, which is useful to develop optical trigger-related functionalities $[19,28]$, and one may think, for instance, in changing between on/off or off/on optical states by simply varying the associated resistance. Figures 5(a) and 5(b) show, respectively, the $I-V$ characteristics and EL- $V$ for four different resistances in series. The data presented for $0 \Omega$ (black circles) and $1 \mathrm{k} \Omega$ (green circles) were obtained experimentally. The effects of intermediary values of the resistance can be simulated by using Kirchhoff's law and the experimental $I(V)$ function without resistance association as the starting point. In this case, the total bias is defined by $V_{\mathrm{ext}}=V+I(V) R$, and the results for $R=100$ and $R=220 \Omega$ are displayed as red and blue circles, respectively, in Figs. 5(a) and 5(b).

The direction of the voltage sweep has been indicated with arrows in Fig. 5. Note that for $R=100 \Omega$, the bistable course of the luminescence changes the light state in the ratio of $\sim 1: 400$ right after the resonance, and the EL bistability follows the same current path. However, for $R=1000 \Omega$, the EL bistability is inverted with respect to the current, and the measured on/off ratio has grown up to 2000:1. Figure 6 displays the calculated optical on-off ratio for a set of simulated resistances from 0 up to $1 \mathrm{k} \Omega$ by using Kirchhoff's law where the conditions for direct and inverted ratios can be assessed. The ratio is increased with higher resistances until an inversion point at $R_{i}=220 \Omega$, and then it goes up to $\sim 10000: 1$. This means that we can, in principle, modulate up to seven orders the light emission enhancement by changing just the resistance. The experimental on-off ratio with $1-\mathrm{k} \Omega$ resistance association is $\sim 2000: 1$. Possible reasons for this discrepancy where the simulated ratios appear overestimated are as follows: a nonlinear internal resistance or a sensitivity increase due to the high resistance, caused by noise or fluctuations. Nevertheless, our results clearly show the modulation of the emitted light by six orders of magnitude. 


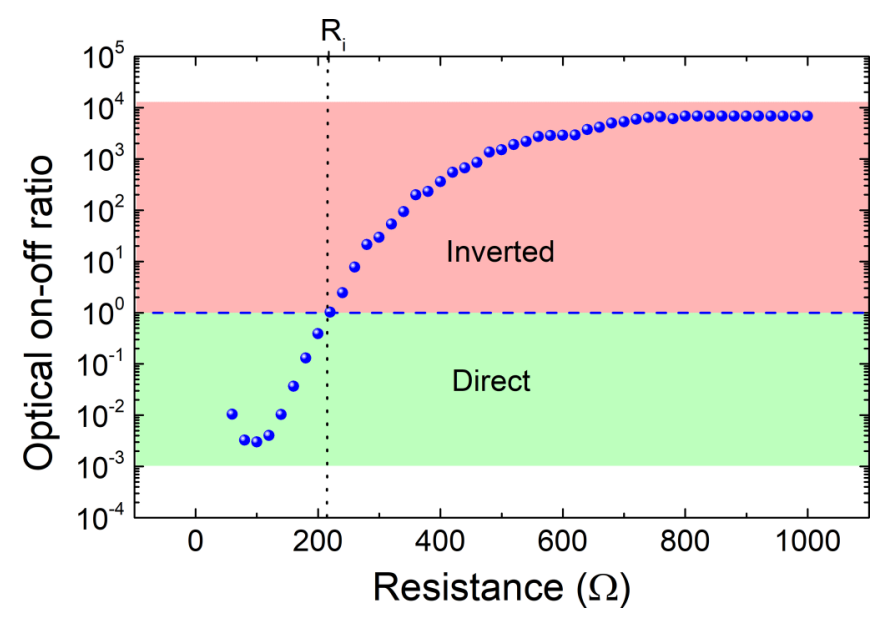

FIG. 6. Simulated optical on-off ratio versus series resistance. The green and red regions represent direct and inverted switches, respectively, compared to the current-voltage transition. The resistance at which the inversion occurs is $R_{i}=220 \Omega$.

\section{CONCLUSIONS}

The correlation between the coherent and incoherent transport channels and the hole generation rate has been assessed in a standard purely $n$-doped GaAs/AlGaAs-based RTD. Each of the coherent and incoherent current channels has been proven to contribute with independent impact ionization processes. Electrons being transported through the incoherent channel undergo a relatively high-energy loss due to lattice scattering. A series resistance has been successfully used as a simple yet effective tool to tune both the current and the electroluminescence emission versus inducing bistable states. By simulating a resistance variation over the $I-V$ characteristics we have shown that it is possible to tune the intensity of the emission by up to six orders of magnitude, and the on-off switch can be either direct or inverted compared to the transport on-off states. These EL properties, such as high emission and tunability, could be useful for trigger-related and optical logic devices.

\section{ACKNOWLEDGMENTS}

The authors gratefully acknowledge financial support from the following agencies: FAPESP (Grants No. 2013/18719-1, No. 2014/07375-2, No. 2014/19142-2, and No. 2014/021123), BAYLAT-FAPESP (Grant No. 2016/50080-9), CNPq (Grants No. 141793/2015-5 and No. 471191/2013-2), CAPES (Grant No. 88881.133567/2016-01), and the German Ministry of Education and Research (BMBF) within the National Project HIRT (Project No. FKZ 13XP5003B).
[1] R. Tsu and L. Esaki, Appl. Phys. Lett. 22, 562 (1973).

[2] L. L. Chang, L. Esaki, and R. Tsu, Appl. Phys. Lett. 24, 593 (1974).

[3] M. Feiginov, H. Kanaya, S. Suzuki, and M. Asada, Appl. Phys. Lett. 104, 243509 (2014).

[4] D. Bae, J. Park, M. Kim, Y. Jeong, and K. Yang, in Proceedings of the Compound Semiconductor Week (CSW) [Includes 28th International Conference on Indium Phosphide Related Materials (IPRM) 43rd International Symposium on Compound Semiconductors (ISCS)], Toyama, Japan, 2016 (IEEE, Piscataway, NJ, 2016).

[5] F. Hartmann, F. Langer, D. Bisping, A. Musterer, S. Höfling, M. Kamp, A. Forchel, and L. Worschech, Appl. Phys. Lett. 100, 172113 (2012).

[6] A. Pfenning, F. Hartmann, M. R. S. Dias, L. K. Castelano, C. Süßmeier, F. Langer, S. Höfling, M. Kamp, G. E. Marques, L. Worschech et al., ACS Nano 9, 6271 (2015).

[7] W. Wei, S. Hao, T. Teng, and S. Xiaowei, J. Semicond. 33, 124002 (2012).

[8] N. Oshima, K. Hashimoto, S. Suzuki, and M. Asada, Electron. Lett. 52, 1897 (2016).

[9] R. Izumi, S. Suzuki, and M. Asada, in Proceedings of the 42nd International Conference on Infrared, Millimeter, and Terahertz Waves (IRMMW-THz), Cancun, Mexico, 2017 (IEEE, Piscataway, NJ, 2017), pp. 1-2.

[10] F. Hartmann, A. Pfenning, M. Rebello Sousa Dias, F. Langer, S. Höfling, M. Kamp, L. Worschech, L. K. Castelano, G. E. Marques, and V. Lopez-Richard, J. Appl. Phys. 122, 154502 (2017).
[11] B. Romeira, L. M. Pessoa, H. M. Salgado, C. N. Ironside, and J. M. L. Figueiredo, Sensors 13, 9464 (2013).

[12] C. R. H. White, M. S. Skolnik, L. Eaves, and M. L. Leadbeater, Appl. Phys. Lett. 58, 1164 (1991).

[13] H. Sheng and S. J. Chua, Opt. Quantum Electron. 26, 1033 (1994).

[14] T. A. Growden, W. Zhang, E. R. Brown, D. F. Storm, K. Hansen, P. Fakhimi, D. J. Meyer, and P. R. Berger, Appl. Phys. Lett. 112, 033508 (2018).

[15] T. A. Growden, W. Zhang, E. R. Brown, D. F. Storm, D. J. Meyer, and P. R. Berger, Light: Sci. Appl. 7, 17150 (2018).

[16] Z. C. Yan, E. Goovaerts, C. Van Hoof, A. Bouwen, and G. Borghs, Phys. Rev. B 52, 5907 (1995).

[17] F. Teran, M. D. Martín, J. M. Calleja, L. Viña, L. Eaves, and M. Henini, Europhys. Lett. 85, 67010 (2009).

[18] V. Schwegler, S. S. Schad, C. Kirchner, M. Seyboth, M. Kamp, K. J. Ebeling, V. E. Kudryashov, A. N. Turkin, A. E. Yunovich, U. Stempfle et al., Phys. Status Solidi A 176, 783 (1999).

[19] M. H. Jiang, X. B. Wang, Q. Xu, M. Li, D. H. Niu, X. Q. Sun, F. Wang, Z. Y. Li, and D. M. Zhang, Opt. Mater. 75, 26 (2018).

[20] C. J. Goodings, H. Mizuta, and J. R. A. Cleaver, J. Appl. Phys. 75, 2291 (1994).

[21] R. Redmer, J. R. Madureira, N. Fitzer, S. M. Goodnick, W. Schattke, and E. Schöll, J. Appl. Phys. 87, 781 (2000).

[22] J. Singh, Electronic and Optoelectronic Properties of Semiconductor Structures (Cambridge University Press, Cambridge, U.K., 2007).

[23] M. Heiblum and M. V. Fischetti, IBM J. Res. Develop. 34, 530 (1990). 
[24] G. Ternent and D. J. Paul, IEEE Trans. Electron Devices 59, 3555 (2012).

[25] J. J. L. Rascol, K. P. Martin, R. E. Carnahan, R. J. Higgins, L. A. Cury, J. C. Portal, B. G. Park, E. Wolak, K. L. Lear, and J. S. Harris, Appl. Phys. Lett. 58, 1482 (1991).
[26] J. N. Schulmann, H. H. de Los Santos, and D. H. Chow, IEEE Electron Device Lett. 17, 220 (1996).

[27] C. Rauch, G. Strasser, M. Kast, and E. Gornik, Superlattices Microstruct. 25, 47 (1999).

[28] H. Hu, X. Zhang, and S. Zhao, Cogent Phys. 4, 1388156 (2017). 